\title{
Varlitinib Downregulates HER/ERK Signaling and Induces Apoptosis in Triple Negative Breast Cancer Cells
}

\author{
Chun-Yu Liu ${ }^{1,2,3,4, *}$, Pei-Yi Chu ${ }^{5,6}$, Chun-Teng Huang ${ }^{2,7}$, Ji-Lin Chen ${ }^{1}$, Hsiu-Ping Yang ${ }^{2}$, \\ Wan-Lun Wang ${ }^{1}$, Ka-Yi Lau ${ }^{1,2}$, Chia-Han Lee ${ }^{1,2}$, Tien-Yun Lan 1,2, Tzu-Ting Huang 1,2 ${ }^{\mathbb{D}}$, \\ Po-Han Lin ${ }^{8,9}$, Ming-Shen Dai ${ }^{10}$ and Ling-Ming Tseng ${ }^{1,2,11}$ \\ 1 Comprehensive Breast Health Center, Taipei Veterans General Hospital, No.201, Sec.2, Shih-Pai Rd., \\ Taipei 112, Taiwan; jlchen_@outlook.com (J.-L.C.); rabbitwang78tw@gmail.com (W.-L.W.); \\ cayeelau@gmail.com (K.-Y.L.); gahanleeo@gmail.com (C.-H.L.); pj165210@gmail.com (T.-Y.L.); \\ abby-eve@hotmail.com (T.-T.H.); lmtseng@vghtpe.gov.tw (L.-M.T.) \\ 2 Division of Medical Oncology, Department of Oncology, Taipei Veterans General Hospital, No.201, Sec.2, \\ Shih-Pai Rd., Taipei 112, Taiwan; huangchunteng@gmail.com (C.-T.H.); synthia730513@gmail.com (H.-P.Y.) \\ 3 School of Medicine, National Yang-Ming University, No.155, Sec.2, Li-Nong Street, Taipei 112, Taiwan \\ 4 Division of Transfusion Medicine, Department of Medicine, Taipei Veterans General Hospital, No.201, Sec.2, \\ Shih-Pai Rd., Taipei 112, Taiwan \\ 5 Department of Pathology, Show Chwan Memorial Hospital, No.542, Sec.1, Chung-Shan Rd., \\ Changhua City 500, Taiwan; chu.peiyi@msa.hinet.net \\ 6 School of Medicine, Fu Jen Catholic University, No.510, Zhong-zheng Rd., Xin-zhuang Dist., \\ New Taipei City 24205, Taiwan \\ 7 Division of Hematology and Oncology, Department of Medicine, Yang-Ming Branch of Taipei City Hospital, \\ No.145, Zhengzhou Rd., Datong Dist., Taipei 103, Taiwan \\ 8 Department of Medical Genetics, National Taiwan University Hospital, No.7, Chung-Shan South Rd., \\ Taipei 100, Taiwan; pohanlin01@gmail.com \\ 9 Graduate institute of medical genomics and proteomics, National Taiwan University, No.1, Sec.4, \\ Roosevelt Rd., Taipei 106, Taiwan \\ 10 Hematology/Oncology, Tri-Service General Hospital, National Defense Medical Center, No.325, Sec.2, \\ Cheng-gong Rd., Taipei 114, Taiwan; dms1201@gmail.com \\ 11 Department of Surgery, Taipei Veterans General Hospital, Taipei 112, Taiwan \\ * Correspondence: cyliu3@vghtpe.gov.tw
}

Received: 29 December 2018; Accepted: 15 January 2019; Published: 17 January 2019

\begin{abstract}
Triple-negative breast cancer (TNBC) is a complex disease associated with the aggressive phenotype and poor prognosis. TNBC harbors heterogeneous molecular subtypes with no approved specific targeted therapy. It has been reported that HER receptors are overexpressed in breast cancer including TNBC. In this study, we evaluated the efficacy of varlitinib, a reversible small molecule pan-HER inhibitor in TNBC. Our results showed that varlitinib reduced cell viability and induced cell apoptosis in most TNBC cell lines but not in MDA-MB-231 cells. MEK and ERK inhibition overcame resistance to varlitinib in MDA-MB-231 cells. Varlitinib inhibited HER signaling which led to inhibition of migration, invasion and mammosphere formation of TNBC cells as well as significant suppression of tumor growth of MDA-MB-468 xenograft mouse model. In summary, these results suggest that HER signaling plays an important role in TNBC progression and that pan-HER inhibition is potentially an effective treatment for TNBC patients.
\end{abstract}

Keywords: pan-HER inhibitor; triple-negative breast cancer 


\section{Introduction}

Triple-negative breast cancer (TNBC) is well-known for its aggressive phenotype and poor prognosis with genetic, transcriptomic and histological heterogeneity [1]. Due to the lack of estrogen receptor (ER), progesterone receptor (PR) and human epidermal growth factor receptor 2 (HER2) amplification, TNBC patients do not respond to hormonal- and trastuzumab-based targeted therapies. Current standard treatment for patients with early-stage TNBC includes cytotoxic chemotherapy with taxane- and anthracycline-based combination therapy, but the prognosis is poor [2]. Several clinical studies reported that platinum-based agents used as the first-line of treatment for patients with TNBC resulted in longer progression-free survival, but eventually also led to the development of drug resistance [3,4]. There has been no targeted therapy approved for TNBC. The heterogeneous molecular subtypes of TNBC present an opportunity for identification of novel therapeutic avenues.

The human HER receptor family has four receptor tyrosine kinases: EGFR (HER1), HER2, HER3 and HER4. HER receptor comprises of an extracellular ligand binding domain, a transmembrane domain, as well as an intracellular protein kinase domain and carboxyl terminal tail [5]. Activation of HER signaling results in mitogen activated protein kinase (MAPK) and phosphoinositide 3-kinase (PI3K)/Akt pathways upregulation, which regulate several cellular processes such as proliferation, apoptosis and metastasis [6]. It has been reported that expression levels of HER family in TNBC are diverse but HER2, HER3 and HER4 transcripts in TNBC are significantly lower than non-TNBC [7]. Nakai et al. reviewed that EGFR expression range from 13\% to $76 \%$ in TNBC. EGFR overexpression was correlated with poor prognosis and drug resistance [8]. Approximately $41.2 \%$ of TNBC cohort was positive staining for HER3, which linked poorer disease free survival (DFS) and overall survival than HER3-negative TNBC subset [9]. HER4 expression has also been reported to be associated with poor prognosis in TNBC [7]. Pan-HER inhibition is considered to be a potential treatment for solid tumor patients including breast, biliary, colorectal, and non-small cell lung cancer [10-12]. Several pan-HER inhibitors such as afatinib, dacomitinib, and neratinib have shown promising results in TNBC in vitro [13-15]. All of these molecules are irreversible inhibitors of HER receptors and have been observed with a relatively high incidence of GI side effects such as diarrhea [16].

Varlitinib (formally known as ASLAN001) is a highly potent, reversible, oral nanomolar small molecule pan-HER inhibitor of the receptor tyrosine. Both in vitro and in vivo experiments have been conducted to demonstrate varlitinib's efficacy [17]. In addition, two varlitinib clinical trials in patients with HER2 positive metastatic breast cancer have been conducted and showed signs of clinical activity [18] (ClinicalTrials.gov Identifier: NCT02338245 and NCT02396108). Given the possible link between TNBC and HER signaling, we sought to study the activity of varlitinib in TNBC cell lines, to determine whether this could be a novel therapeutic opportunity.

\section{Results}

\subsection{Varlitinib Exerts Anti-Proliferation Ability and Induces Apoptosis}

We first examined the endogenous expression levels of HER receptors in TNBC cell lines, HER2-amplified SK-BR-3 cells as well as MCF 10A breast epithelial cell line. Results of western blot analysis showed that most of TNBC cell lines expressed higher levels of EGFR and HER3 than MCF 10A cells. TNBC cell lines were deficient in HER2 and differentially expressed HER4 (Figure 1A). To explore varlitinib's anti-tumor activity, cells were treated with varlitinib at various concentrations. Compared with MCF 10A cell lines, most of the cell lines exhibited lower $\mathrm{IC}_{50}$ except MDA-MB-231 cells (Figure 1B and Figure S1). Moreover, varlitinib significantly induced cell apoptosis in MDA-MB-453 and MDA-MB-468 cells but not in MDA-MB-231 cells (Figure 1B,C). 
A

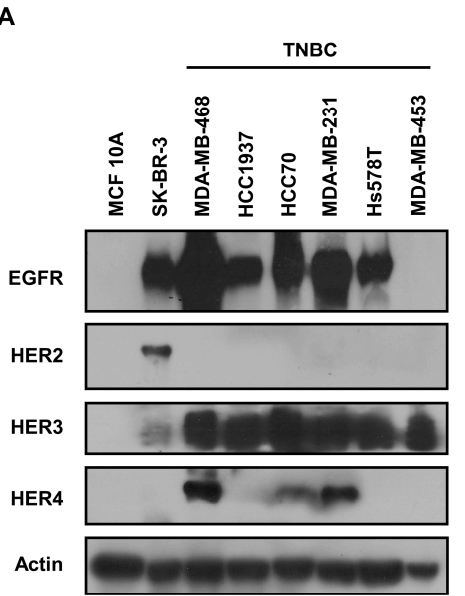

B

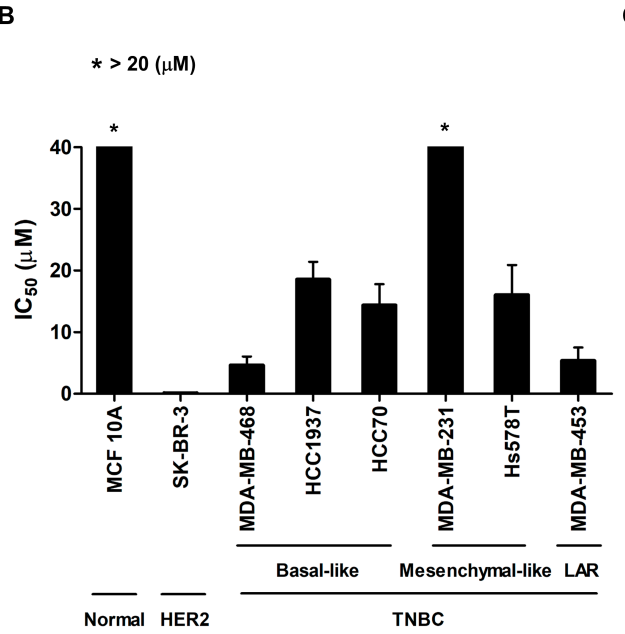

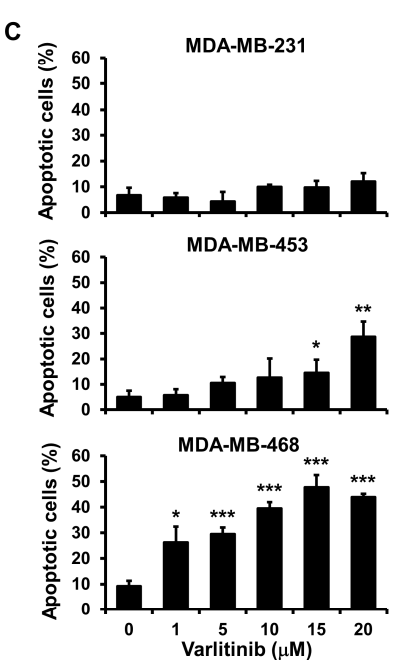

Figure 1. Varlitinib exerts anti-proliferation ability and induces apoptosis in MDA-MB-453 and MDA-MB-468 cells but not MDA-MB-231 cells. (A) Whole-cell extracts were analyzed by western blot analysis using antibodies against anti-EGFR, anti-HER2, anti-HER3, anti-HER4 and anti- $\beta$-actin. (B) Cells were treated with various concentrations of varlitinib for $72 \mathrm{~h}$ for MTT assay. $\mathrm{IC}_{50}$ values were determined and shown. (C) MDA-MB-231, MDA-MB-453 and MDA-MB-468 cells were treated with various concentrations of varlitinib for $72 \mathrm{~h}$. The treated cells were analyzed using flow cytometry. The means \pm SEM of three independent experiments performed in triplicate are shown. Student's $t$-test, ${ }^{*} p<0.05,{ }^{* *} p<0.01,{ }^{* * *} p<0.001$.

\subsection{Varlitinib Suppresses MEK/ERK Pathway in TNBC Cells}

To explore the clinical significance of HER family, we examined data from The Cancer Genome Atlas (TCGA) database. EGFR, HER2, HER3 and HER4 gene alterations, including copy number variation, mutation and mRNA dysregulation, in patients with breast cancer and TNBC, were analyzed. Results showed that most of TNBC patients harbored EGFR upregulation compared to HER2, HER3 and HER4 (Figure S2A). In addition, TNBC tumor tissues harbored higher pEGFR $^{\mathrm{Y} 1173}$ and $\mathrm{pHER}^{\mathrm{Y} 1289}$ than normal tissues (Figure S2B). To evaluate the pan-HER inhibitor capacity in vitro, we examined the phosphorylation of HER family in varlitinib-treated SK-BR-3 (a HER2-expressing breast cancer cell line) and MDA-MB-468 cells. Data showed varlitinib reduced pEGFR, pHER3 and pHER4 in MDA-MB-468 cells as well as reduced pHER2 in SK-BR-3 cells (Figure 2A,B). Activation of HER receptors leads to the activation of downstream pathways including RAS/RAF/MEK/ERK and PI3K/Akt signaling. Our western blot results demonstrated that varlitinib treatment inhibited EGFR, AKT, MEK and ERK activation in MDA-MB-453 and MDA-MB-468 cells. In addition, varlitinib treatment also resulted in increased levels of cleaved PARP and cleaved Caspase- 3 in these TNBC cell lines. Conversely, varlitinib did not inhibit MEK/ERK signaling in MDA-MB-231 cells (Figure 2C). 
A

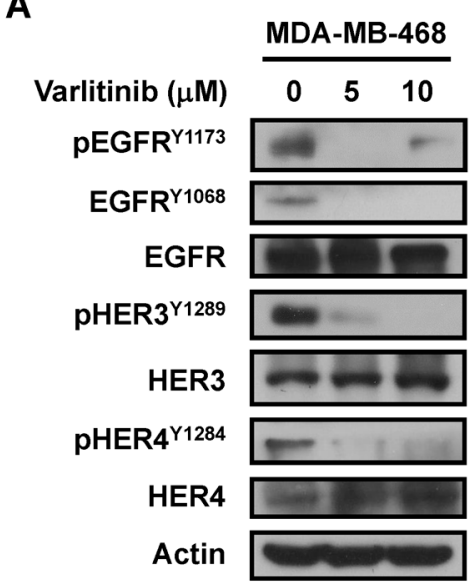

B

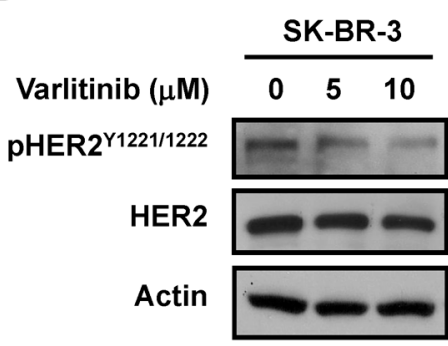

C

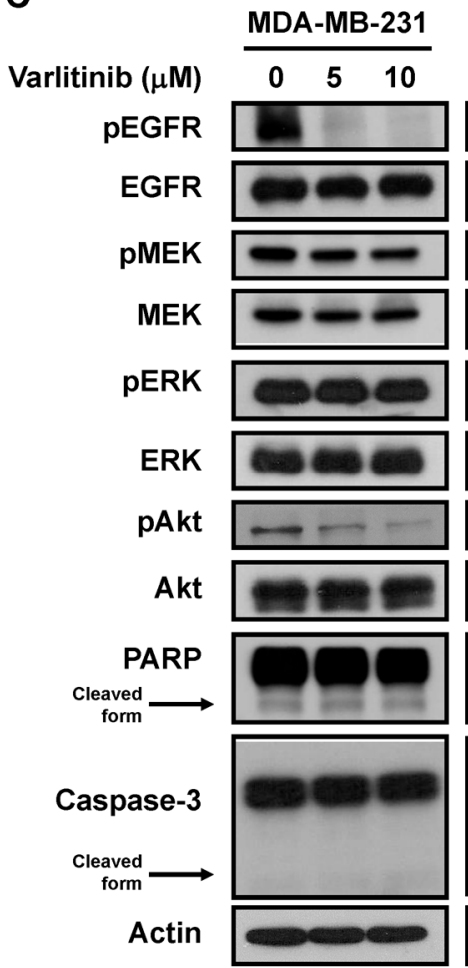

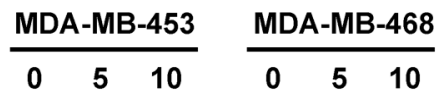

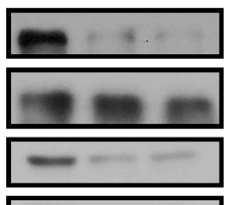

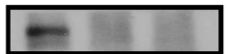

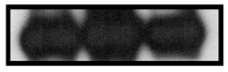

E.
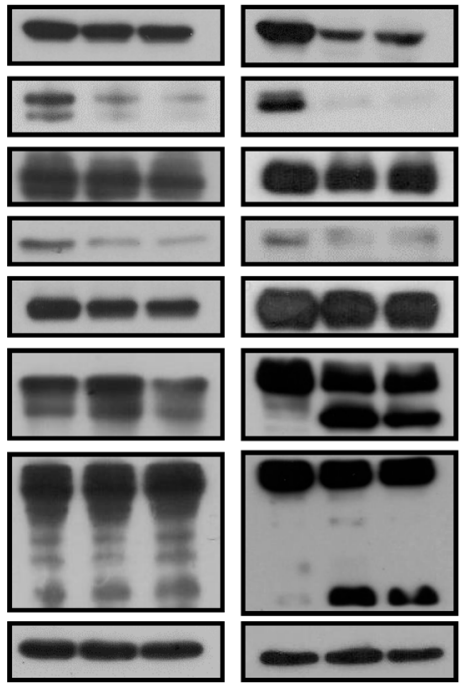

Figure 2. Varlitinib inhibits MEK/ERK and Akt pathway in TNBC cells. (A,B) Whole-cell extracts of SK-BR-3 and MDA-MB-468 cells treated with indicated concentration of varlitinib for $48 \mathrm{~h}$ were prepared for western blot analysis using antibodies against anti-phospho-EGFR ${ }^{\mathrm{Y} 1173}$, anti-phosphoEGFR $^{\mathrm{Y} 1068}$, anti-EGFR, anti-phospho-HER2 ${ }^{\mathrm{Y} 1221 / 1222}$, anti-HER2, anti-phospho-HER3 ${ }^{\mathrm{Y} 1289}$, anti-HER3, anti-phospho-HER4 ${ }^{\mathrm{Y} 1284}$, anti-HER4 and anti- $\beta$-actin. (C) The whole-cell extracts from MDA-MB-231, MDA-MB-453 and MDA-MB-468 cells treated with indicated concentration of varlitinib for $48 \mathrm{~h}$ were prepared for western blot analysis using antibodies against anti-phospho-EGFR, anti-EGFR, anti-phospho-MEK, anti-MEK, anti-phospho-ERK, anti-ERK, anti-phospho-Akt, anti-Akt, anti-PARP, anti-Caspase- 3 and anti- $\beta$-actin.

\subsection{Varlitinib Induces Apoptosis through ERK Inhibition in TNBC Cells}

RAF/MEK/ERK pathway has different effects on prevention of apoptosis, cell cycle arrest and induction of drug resistance [19]. Our results suggested that MEK/ERK activation play a role in resistance to varlitinib-induced apoptosis in MDA-MB-231 cells. MDA-MB-231 cells were treated with varlitinib in combination with either MEK inhibitor or ERK inhibitor. Results showed that varlitinib combination treatment with MEK/ERK inhibitors induced apoptosis and also increased the level of cleaved PARP in MDA-MB-231 cells (Figure 3A,B). In contrast, varlitinib-induced cell apoptosis or PARP cleavage were rescued by ERK2 overexpression but not ERK1 overexpression in MDA-MB-468 cells (Figure 3C). 
A

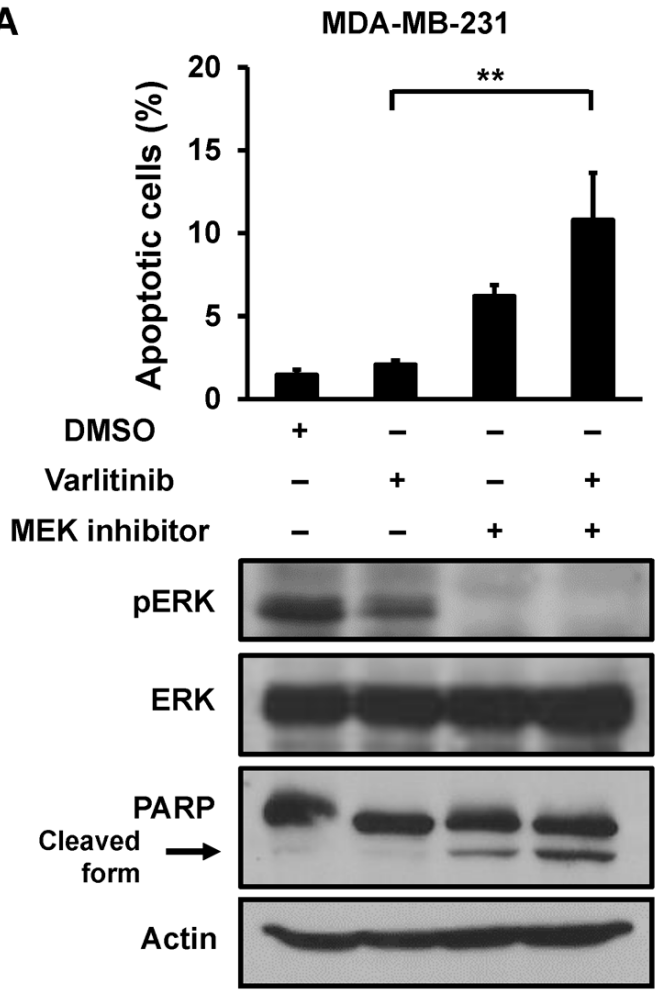

C

MDA-MB-468

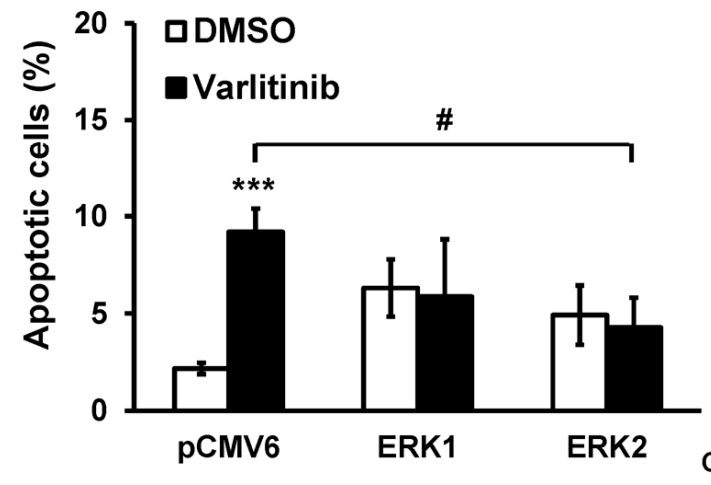

B

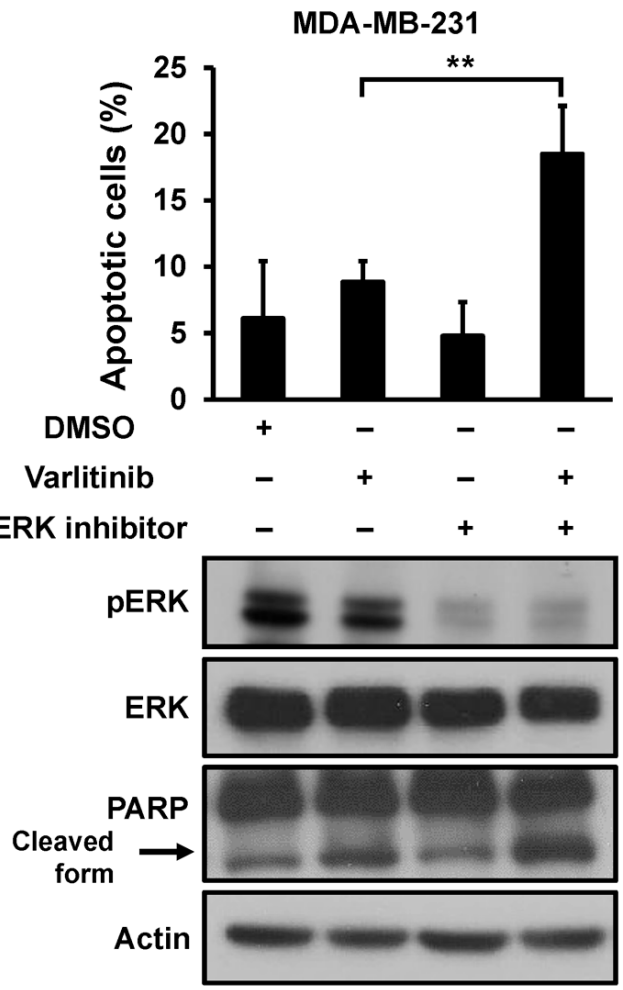

MDA-MB-468

$\begin{array}{ccccccc}\text { PCMV6 } & + & + & - & - & - & - \\ \text { ERK1 } & - & - & + & + & - & - \\ \text { ERK2 } & - & - & - & - & + & + \\ \text { DMSO } & + & - & + & - & + & - \\ \text { Varlitinib } & - & + & - & + & - & +\end{array}$

PERK

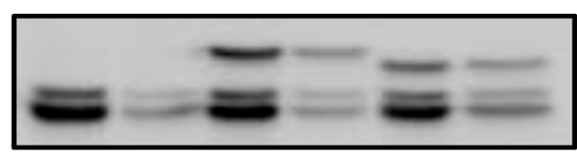

ERK

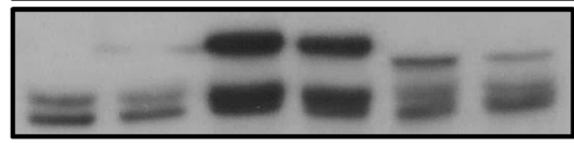

PARP

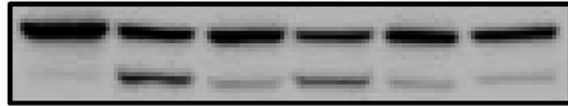

Actin

Figure 3. ERK signaling mediates varlitinib-induced apoptosis in TNBC cells. (A) MDA-MB-231 cells were treated with MEK inhibitor GDC-0973, varlitinib or DMSO for 48 h. (B) MDA-MB-231 cells were treated with ERK inhibitor SCH772984, varlitinib or DMSO for 48 h. (C) MDA-MB-468 cells were transfected with ERK1-, ERK2-expressing or control plasmids (pCMV6) for $24 \mathrm{~h}$, and the transfected cells were further treated with varlitinib or DMSO for $48 \mathrm{~h}$. Apoptosis in the treated cells was analyzed by flow cytometry (left) and the whole-cell extracts of treated cells were analyzed by western blot analysis using antibodies against anti-phospho-ERK, anti-ERK, anti-PARP and anti- $\beta$-actin (right). The means \pm SEM of three independent experiments performed in triplicate are shown. Student's $t$-test, ${ }^{* *} p<0.01,{ }^{* * *} p<0.001$ compared with cells treated with DMSO control. \# $p<0.05$ compared with cells transfected with pCMV6 vector and treated with varlitinib. 


\subsection{Varlitinib Inhibits Migration, Invasion and Mammosphere Formation of TNBC Cells}

To evaluate the role of varlitinib in cancer progression of TNBC cells, functional assays were performed. Our results showed that varlitinib treatment inhibited cell migration, invasion and mammosphere formation of MDA-MB-231 and MDA-MB-468 cells (Figure 4A-C).
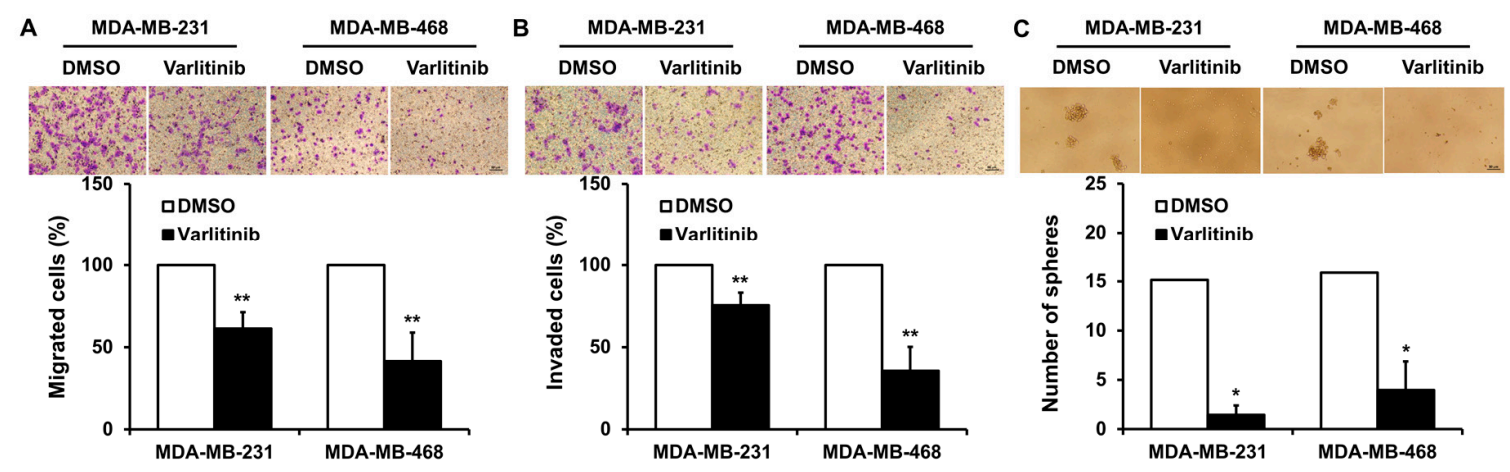

Figure 4. Varlitinib reduces the abilities of migration, invasion and mammosphere formation of TNBC cells. (A-C) MDA-MB-231 and MDA-MB-468 cells were treated with varlitinib or DMSO for subsequent migration (A), invasion (B) and mammosphere assays (C). The means \pm SEM of three independent experiments performed in triplicate are shown $(100 \times$ magnification times for $A, B, C)$. Student's $t$-test, ${ }^{*} p<0.05,{ }^{* *} p<0.01$.

\subsection{Varlitinib Shows Anti-Tumor Effect in TNBC Xenograft Model}

Nude mice were subcutaneously implanted with MDA-MB-468 cells to evaluate anti-tumor activity of varlitinib. Once xenograft tumor sizes reached $200 \mathrm{~mm}^{3}$ varlitinib were orally administered. Varlitinib suppressed tumor growth in MDA-MB-468 xenograft mice with no effect on body weight (Figure 5A-C). In comparison to the control group, varlitinib treatment significantly suppressed EGFR and ERK activation with increased PARP cleavage (Figure 5D). Immunohistochemical staining was performed to further examine protein expression and localization within the xenograft tumors. The results showed that varlitinib treatment reduced EGFR and ERK phosphorylation and elicited cell apoptosis with M30 staining (Figure 5D,E). These results demonstrated varlitinib exerting anti-tumor activity in TNBC via the inhibition of HER receptor and downstream signaling. 
A

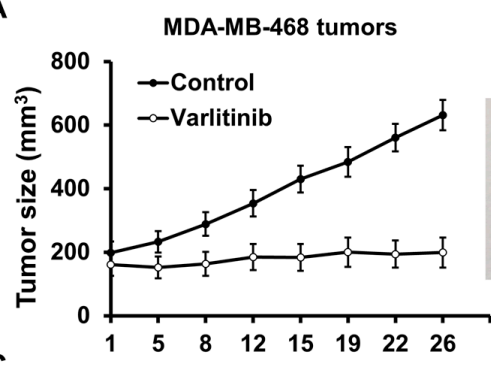

C

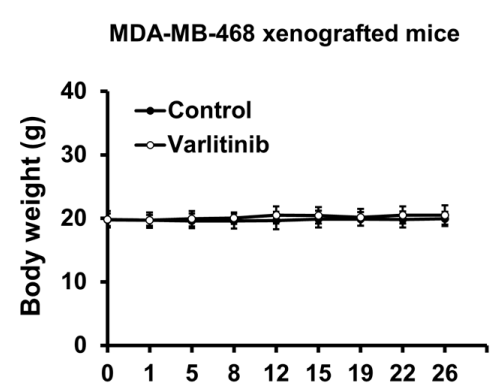

B

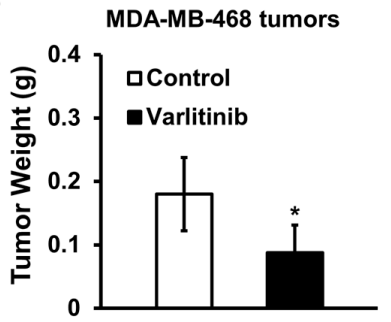

D

MDA-MB-468 tumors

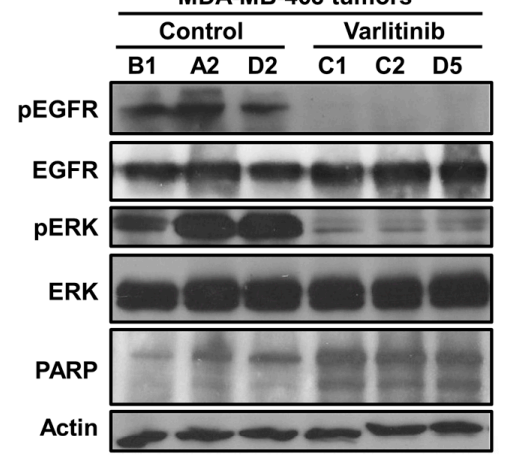

E

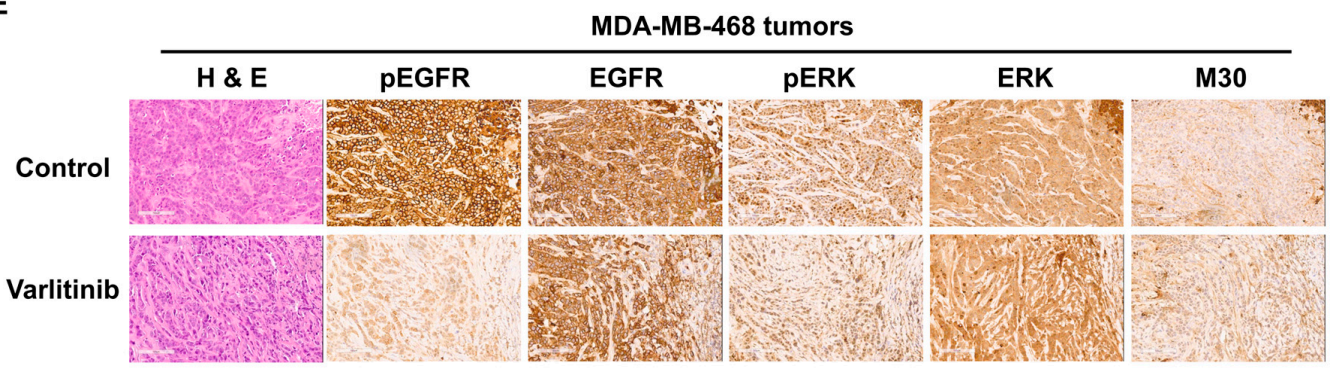

Figure 5. Varlitinib inhibits tumor growth of TNBC. (A-E) MDA-MB-468-bearing mice were treated with varlitinib at $100 \mathrm{mg} / \mathrm{kg}$ BID or vehicle and the (A) xenografted tumors sizes, (B) weights and (C) the body weights of xenografted mice were measured. (D) The xenografted tumors were analyzed by western blotting using antibodies against anti-pEGFR, anti-EGFR, anti-pERK, anti-ERK, anti-PARP and anti- $\beta$-actin. (E) Immunohistochemical staining of pEGFR, EGFR, pERK, ERK and M30 in MDA-MB-468 xenografts $\left(200 \times\right.$ magnification times for E). Student's $t$-test, ${ }^{*} p<0.05$. Data are shown as mean $\pm \mathrm{SD}$.

\section{Discussion}

HER receptors play a crucial role in breast cancer tumorigenesis including TNBC. EGFR, HER3 and HER4 expression in TNBC patients was associated with poor DFS $[7,9,20]$. These receptors might serve as prognostic markers and targets for treatments. However, several clinical studies demonstrated that EGFR inhibitors monotherapy and chemotherapy combination showed a partial response in TNBC [21,22]. The HER receptors form homodimers and heterodimers. Hetero-dimerization of EGFR with HER2, HER3 or HER4 might limit anti-tumor effect of EGFR inhibition, and pan-HER inhibitor could improve the efficacy of EGFR inhibition through limiting receptor cross-talk signaling [23].

Pan-HER inhibitors include afatinib, dacomitinib, and neratinib which induce irreversible inhibition [13,24]. U.S. Food and Drug Administration (FDA) approved afatinib for patients with metastatic non-small cell lung cancer whose tumors express EGFR exon 19 deletions or exon 21 (L858R) substitution mutations as well as the patients with advanced squamous cell lung cancer who have progressed after treatment with platinum-based chemotherapy [15]. In addition, FDA approved neratinib as extended adjuvant treatment for patients with early stage HER2 positive breast cancer. It has been reported that afatinib and neratinib inhibited cell growth of TNBC [25,26], however, TNBC patients have not shown significant improvement in clinical trials with these inhibitors [14,27]. 
The anti-tumor effects of varlitinib in TNBC were investigated in vitro and in vivo. From our experimental results, varlitinib suppressed cell viability in most of TNBC cells, however, MDA-MB-231 cells were resistant to apoptotic effect of varlitinib (Figure 1). Varlitinib is a reversible pan-HER inhibitor with nanomolar potency against HER1, HER2 and HER4 in cell-based assays of gastric cancer [17]. HER3 has defective tyrosine kinase activity. Upon hetero-dimerization, HER3 can be phosphorylated by EGFR or HER2. Therefore, a pan-HER inhibitor could also inhibit HER3 receptor indirectly [13]. Our data also indicated that varlitinib exhibited pan-HER inhibitor activity in breast cancer cells. HER signaling triggers RAS/RAF/MEK/ERK and PI3K/Akt signal pathways. We examined the downstream signaling and found that varlitinib inhibited activation of MEK and ERK in MDA-MB-453 and MDA-MB-468 but not in MDA-MB-231 cells (Figure 2).

In MDA-MB-468 cells, it was also shown that reactivation of ERK in combine with varlitinib repressed the cell apoptosis effect of varlitinib. It was shown that the combination of MEK or ERK inhibitors with varlitinib induced cell apoptosis in MDA-MB-231 cells (Figure 3). MDA-MB-231 cells harbored BRAF ${ }^{\mathrm{G} 464 \mathrm{~V}}$ and KRAS ${ }^{\mathrm{G} 13 \mathrm{D}}$ mutations [28]. One study showed that exogenous expression of BRAF mutants including BRAF ${ }^{G 464 V}$ in SK-BR-3 cells treated with lapatinib, a HER1/HER2 tyrosine kinase inhibitor, did not reduce pERK level [29]. This may possibly be due to the drug resistance associated with KRAS mutations. Another study showed KRAS ${ }^{\mathrm{G} 13 \mathrm{D}}$ and KRAS ${ }^{\mathrm{G} 12 R}$ mutations in drug resistance to cetuximab in vitro and in vivo [30,31]. These studies indicated that KRAS and BRAF mutations drove ERK dependent growth in various cancers. RAS/RAF/MEK/ERK pathway is activated by upstream molecules alteration. Mutations occurring in this pathway might lead to chemoresistance. Targeting this cascade is complicated and the presence of upstream mutations may result in pathway activation [32,33]. However, BRAF and KRAS mutations are rare in breast cancer [34] with BRAF and KRAS mutated in $0.45 \%$ and $0.54 \%$ of The Cancer Genome Atlas (TCGA) breast cancer genomes respectively.

Functional assays showed that varlitinib led to inhibition of migration, invasion and mammosphere formation in TNBC cells (Figure 4) regardless of their MEK and ERK status. Varlitinib inactivated Akt in TNBC cells shown in Figure 2. Several studies revealed that Akt pathway participated in cancer metastasis including breast cancer [35]. Akt activation up-regulates Snail, a transcriptional repressor of E-cadherin, leading to epithelial-mesenchymal transition (EMT) [36]. EMT elicits acquisition of motility and invasion and contributes to stemness [37,38]. A phase II LOTUS trial demonstrated that improved progression free survival in patients with metastatic TNBC receiving Akt inhibitor ipatasertib plus paclitaxel [39]. Our result shows varlitinib's anti-tumor activities in both in vitro and in vivo experiments (Figures 4 and 5). Varlitinib may exhibit anti-tumor activities not only through MEK/ERK inhibition but Akt dephosphorylation. However, the role of pan-HER inhibition in tumor metastasis in vivo should be further investigated.

To this date, several irreversible small molecule pan-HER inhibitors are in clinical trials, with diarrhea and skin toxicity as common adverse effects [40]. In general, reversible drugs are considered to be safer than irreversible drugs, and clinical experience with varlitinib shows a low degree of GI side effects. Clinical trial data with varlitinib in MBC patients failing trastuzumab showed promising activity compared to lapatinib [18].

\section{Materials and Methods}

\subsection{Cell Culture, Reagents and Transfection}

Human TNBC cell lines MDA-MB-231, MDA-MB-468, MDA-MB-453, HCC1937 and Hs578T and HER2-positive SK-BR-3 human breast cancer cells were cultured in Dulbecco's Modified Eagle Medium (DMEM) with 10\% fetal bovine serum. Human HCC70 TNBC cell lines and MCF 10A breast epithelial cell line were cultured in RPMI-1640 Medium with $10 \%$ fetal bovine serum. Cell lines were purchased from American Type Culture Collection (Manassas, VA, USA). For transfection, cells were seeded onto 6-well for $24 \mathrm{~h}$ and transiently transfected by Lipofectamine ${ }^{\mathrm{TM}} 3000$ Reagent (Thermo Fisher 
Scientific, Waltham, MA, USA). The MEK inhibitor GDC-0973 (Cobimetinib, Adooq BioScience, Irvine, CA, USA), ERK1/2 inhibitor SCH772984 (Selleck Chemicals, Houston, TX, USA) were used at the indicated concentrations in dimethyl sulfoxide (DMSO). Varlitinib for in vitro and in vivo experiments were generously provided by ASLAN Pharmaceuticals.

\subsection{Western Blot Analysis}

Whole cell extract was prepared using RIPA buffer (Thermo Scientific) with a Halt ${ }^{\mathrm{TM}}$ Protease and Phosphatase Inhibitor Cocktail (Thermo Scientific) [15]. Laemmli's sample buffer was added to the cell lysates and boiled at $95{ }^{\circ} \mathrm{C}$ for $5 \mathrm{~min}$. The cell lysates were then analyzed by sodium dodecyl sulfate-polyacrylamide gel electrophoresis using antibodies against anti-EGF Receptor, anti-phospho-EGF Receptor (Tyr1068), anti-HER2/ErbB2, anti-HER3/ErbB3, anti-HER4/ErbB4, anti-phospho-MEK1/2 (Ser217/221), anti-MEK1/2, anti-phospho-p44/42 MAPK (Erk1/2) (Thr202/Tyr204), anti-p44/42 MAPK (Erk1/2), anti-Akt, anti-phospho-Akt (Ser473), anti-PARP, anti-Caspase- 3 and anti- $\beta$-actin (Cell Signaling).

\subsection{MTT Assay}

Cells were seeded onto 96-well plates for $24 \mathrm{~h}$ and treated with indicated concentrations of varlitinib for $72 \mathrm{~h}$. The treated cells were incubated with $0.5 \mathrm{mg} / \mathrm{mL}$ MTT (Sigma-Aldrich, St. Louis, MO, USA) in each well at $37^{\circ} \mathrm{C}$ for $3 \mathrm{~h}$. The violet MTT formazan precipitates were subsequently dissolved in $100 \mu \mathrm{L}$ DMSO. The absorbance at $570 \mathrm{~nm}$ was measured on an UQuant reader.

\subsection{Flow Cytometry Analysis}

For apoptosis detection, $5 \times 10^{5}$ cells were harvested and suspended in $200 \mu \mathrm{L} 1 \times$ Binding Buffer containing $5 \mu \mathrm{L}$ APC Annexin V (BD Biosciences, San Jose, CA, USA) and $4 \mu \mathrm{L}$ of $0.2 \mathrm{mg} / \mathrm{mL}$ Propidium Iodide (PI). Cells were incubated for $15 \mathrm{~min}$ at room temperature protected from light. The apoptotic cells were measured by APC Annexin V and PI double staining and analyzed by flow cytometry.

\subsection{Migration and Invasion Assays}

The migration and invasion assays were performed in 24-well plate for 16 and $20 \mathrm{~h}$ respectively. $3 \times 10^{4}$ cells in $200 \mu \mathrm{L}$ of serum free medium were seeded onto upper Cell Culture Insert with $8 \mu \mathrm{m}$ pores (Greiner Bio One, Kremsmünster, Austria) for migration assay and Matrigel matrix (Corning, New York, NY, USA) coated Cell Culture Insert for invasion assay respectively. The lower chamber contained $900 \mu \mathrm{L}$ of complete medium. The migrated and invaded cells were fixed with methanol for $10 \mathrm{~min}$ and stained with $0.005 \%$ crystal violet for $1 \mathrm{~h}$ at room temperature. The numbers of migrated and invaded cells were counted under the microscope from 10 random fields.

\subsection{Mammosphere Assay}

The mammosphere formation assay was performed in 96-well Ultra-Low attachment plates (Corning). 500 cells were incubated in DMEM/F-12 medium containing 1\% N-2, 2\% B-27, 20 ng/mL bFGF and $20 \mathrm{ng} / \mathrm{mL}$ EGF (Gibco, Thermo Fisher Scientific) at $37{ }^{\circ} \mathrm{C}$ for 9 days and fresh medium was supplemented every 3 days. The mammospheres which exceed $50 \mu \mathrm{m}$ in diameter were counted under the microscope.

\subsection{Xenograft Tumor Growth}

The animal experiments were approved by the Institutional Animal Care and Use Committee of Taipei Veterans General Hospital (IACUC No. 2017-073). Five-week-old female NCr athymic nude mice were obtained from the National Applied Research Laboratories National Laboratory Animal Center (Taipei, Taiwan). Mice were inoculated subcutaneously in the right flank with MDA-MB-468 
cells $\left(1.5 \times 10^{6}\right)$ suspended in $0.1 \mathrm{~mL}$ serum-free medium containing 30\% matrigel (BD Biosciences) under isoflurane anesthesia. MDA-MB-468 tumor-bearing mice with mean tumor volume $200 \mathrm{~mm}^{3}$ were randomly divided into 2 groups ( $n=10$ per group) and treated with varlitinib at $100 \mathrm{mg} / \mathrm{kg}$ BID $\mathrm{PO}$ or vehicle (solutol HS15:propylene glycol:PBS = 25:25:50). Tumor sizes were measured using caliper and volumes calculated using a standard formula: width ${ }^{2} \times$ length $\times 0.52$. Tumors were harvested from mice at the time of sacrifice for further data analysis. All animal studies were performed in accordance with protocols approved by the Institutional Laboratory Animal Care and Use Committee of the Taipei Veterans General Hospital.

\subsection{Immunohistochemical Staining}

Primary antibodies against anti-pEGFR (ab40815, Abcam, Cambridge, MA, USA), anti-EGFR (Z2037, Zeta Corporation, Sierra Madre, CA, USA), anti-pERK (ab50011, Abcam), anti-ERK (ab17942, Abcam), and anti-M30 CytoDEATH (No. 10700, PEVIVA, West Chester, OH, USA) antibodies were used as previously described [41].

\subsection{Statistical Analysis}

All statistical analyses were performed using GraphPad Prism 5.0 (GraphPad Software, Inc., San Diego, CA, USA). Data analysis was performed using Student's $t$-test, and statistical significance was defined as a $p$-value of less than 0.05 .

\section{Conclusions}

In this study, following the observation that HER based mechanisms may be active in TNBC, our result shows varlitinib is able to suppress activation of ERK and Akt pathways and can exert significant anti-tumor activity in TNBC.

Supplementary Materials: The following are available online at http:/ /www.mdpi.com/2072-6694/11/1/105/s1, Figure S1: Varlitinib suppressed cell viability, Figure S2: Clinical significance of HER family in TNBC.

Author Contributions: Conceptualization, C.-Y.L. and L.-M.T.; Data curation, P.-Y.C., C.-T.H., J.-L.C., T.-T.H., P.-H.L. and M.-S.D.; Methodology, P.-Y.C. and T.-T.H.; Supervision, C.-Y.L., C.-T.H. and L.-M.T.; Validation, P.-Y.C., J.-L.C., H.-P.Y., W.-L.W., K.-Y.L., C.-H.L. and T.-Y.L.; Writing-original draft, C.-Y.L. and J.-L.C. All authors had substantial contributions to the conception or design of the work. All authors read the final manuscript. All authors agreed with the accuracy and integrity of all parts of the work.

Funding: This study is supported by grants from Yang-Ming Branch of Taipei City Hospital (10601-62-020; 10701-62-030); the Yen Tjing Ling Medical Foundation (CI-107-10); the Ministry of Science and Technology, Taiwan (MOST 104-2628-B-075-001-MY3, 105-2314-B-075-044-MY3); Taipei Veterans General Hospital (V106C-101, V107C-025, V107C-016, V106D27-002-MY2-2) and TVGH-NTUH Joint Research Program (VN106-07) from Taipei Veterans General Hospital and National Taiwan-University Hospital and from the Ministry of Health and Welfare, Executive Yuan, Taiwan (MOHW107-TDU-B-212-112015 for the Center of Excellence for Cancer Research at Taipei Veterans General Hospital).

Acknowledgments: The laboratory works were completed using facilities from Medical Science\& Technology Building of Taipei Veterans General Hospital. The authors would like to acknowledge ASLAN Pharmaceuticals for their generous support of varlitinib for in vitro and in vivo experiments.

Conflicts of Interest: The authors declare no potential conflict of interest.

\section{References}

1. Pareja, F.; Geyer, F.C.; Marchio, C.; Burke, K.A.; Weigelt, B.; Reis-Filho, J.S. Triple-negative breast cancer: The importance of molecular and histologic subtyping, and recognition of low-grade variants. NPJ Breast Cancer 2016, 2, 16036. [CrossRef] [PubMed]

2. Yao, H.; He, G.; Yan, S.; Chen, C.; Song, L.; Rosol, T.J.; Deng, X. Triple-negative breast cancer: Is there a treatment on the horizon? Oncotarget 2017, 8, 1913-1924. [CrossRef] [PubMed]

3. Zhang, J.; Fan, M.; Xie, J.; Wang, Z.; Wang, B.; Zhang, S.; Wang, L.; Cao, J.; Tao, Z.; Li, T.; et al. Chemotherapy of metastatic triple negative breast cancer: Experience of using platinum-based chemotherapy. Oncotarget 2015, 6, 43135-43143. [CrossRef] [PubMed] 
4. Bouwman, P.; Jonkers, J. Molecular pathways: How can BRCA-mutated tumors become resistant to PARP inhibitors? Clin. Cancer Res. 2014, 20, 540-547. [CrossRef]

5. Roskoski, R., Jr. The ErbB/HER family of protein-tyrosine kinases and cancer. Pharmacol. Res. 2014, 79, 34-74. [CrossRef]

6. Wieduwilt, M.J.; Moasser, M.M. The epidermal growth factor receptor family: Biology driving targeted therapeutics. Cell. Mol. Life Sci. 2008, 65, 1566-1584. [CrossRef]

7. Kim, J.Y.; Jung, H.H.; Do, I.G.; Bae, S.; Lee, S.K.; Kim, S.W.; Lee, J.E.; Nam, S.J.; Ahn, J.S.; Park, Y.H.; et al. Prognostic value of ERBB4 expression in patients with triple negative breast cancer. BMC Cancer 2016, 16, 138. [CrossRef]

8. Corkery, B.; Crown, J.; Clynes, M.; O’Donovan, N. Epidermal growth factor receptor as a potential therapeutic target in triple-negative breast cancer. Ann. Oncol. 2009, 20, 862-867. [CrossRef]

9. Bae, S.Y.; La Choi, Y.; Kim, S.; Kim, M.; Kim, J.; Jung, S.P.; Choi, M.Y.; Lee, S.K.; Kil, W.H.; Lee, J.E.; et al. HER3 status by immunohistochemistry is correlated with poor prognosis in hormone receptor-negative breast cancer patients. Breast Cancer Res. Treat. 2013, 139, 741-750. [CrossRef]

10. Tebbutt, N.; Pedersen, M.W.; Johns, T.G. Targeting the ERBB family in cancer: Couples therapy. Nat. Rev. Cancer 2013, 13, 663-673. [CrossRef]

11. Nam, H.J.; Kim, H.P.; Yoon, Y.K.; Song, S.H.; Min, A.R.; Han, S.W.; Im, S.A.; Kim, T.Y.; Oh, D.Y.; Bang, Y.J. The irreversible pan-HER inhibitor PF00299804 alone or combined with gemcitabine has an antitumor effect in biliary tract cancer cell lines. Investig. New Drugs 2012, 30, 2148-2160. [CrossRef]

12. Adams, R.; Brown, E.; Brown, L.; Butler, R.; Falk, S.; Fisher, D.; Kaplan, R.; Quirke, P.; Richman, S.; Samuel, L.; et al. Inhibition of EGFR, HER2, and HER3 signalling in patients with colorectal cancer wild-type for BRAF, PIK3CA, KRAS, and NRAS (FOCUS4-D): A phase 2-3 randomised trial. Lancet Gastroenterol. Hepatol. 2018, 3, 162-171. [CrossRef]

13. Wang, X.; Batty, K.M.; Crowe, P.J.; Goldstein, D.; Yang, J.L. The Potential of panHER Inhibition in Cancer. Front. Oncol. 2015, 5, 2. [CrossRef] [PubMed]

14. Feldinger, K.; Kong, A. Profile of neratinib and its potential in the treatment of breast cancer. Breast Cancer 2015, 7, 147-162. [PubMed]

15. Bennouna, J.; Moreno Vera, S.R. Afatinib-based combination regimens for the treatment of solid tumors: Rationale, emerging strategies and recent progress. Future Oncol. 2016, 12, 355-372. [CrossRef]

16. Yang, J.C.; Sequist, L.V.; Zhou, C.; Schuler, M.; Geater, S.L.; Mok, T.; Hu, C.P.; Yamamoto, N.; Feng, J.; O'Byrne, K.; et al. Effect of dose adjustment on the safety and efficacy of afatinib for EGFR mutation-positive lung adenocarcinoma: Post hoc analyses of the randomized LUX-Lung 3 and 6 trials. Ann. Oncol. 2016, 27, 2103-2110. [CrossRef] [PubMed]

17. Kim, J.; Im, S.; Lee, K.; Kim, J.W.; Lee, K.; Han, S.; Kim, T.; Choi, I.S.; Oh, D.; Lee, N.; et al. 664p Phase IIa study to evaluate the biological activity of aslan001 in HER-1/2 co-expressing or her-2 amplified advanced gastric cancer. Ann. Oncol. 2014, 25, iv226. [CrossRef]

18. Lee, S.C.; Chen, S.C.; Dai, M.S.; Lee, G.E.; Liu, C.L.; Chan, A.; Chang, H.K.; Tseng, L.M.; Chay, W.Y.; Chow, L.W.C.; et al. 102PMulticenter phase 2 trial of varlitinib versus lapatinib in combination with capecitabine in patients with HER2+ metastatic breast cancer (MBC) who failed prior trastuzumab therapy. Ann. Oncol. 2017, 28, mdx654.010. [CrossRef]

19. McCubrey, J.A.; Steelman, L.S.; Chappell, W.H.; Abrams, S.L.; Wong, E.W.; Chang, F.; Lehmann, B.; Terrian, D.M.; Milella, M.; Tafuri, A.; et al. Roles of the Raf/MEK/ERK pathway in cell growth, malignant transformation and drug resistance. Biochim. Biophys. Acta 2007, 1773, 1263-1284. [CrossRef]

20. Nakai, K.; Hung, M.C.; Yamaguchi, H. A perspective on anti-EGFR therapies targeting triple-negative breast cancer. Am. J. Cancer Res. 2016, 6, 1609-1623.

21. Harding, J.; Burtness, B. Cetuximab: An epidermal growth factor receptor chemeric human-murine monoclonal antibody. Drugs Today 2005, 41, 107-127. [CrossRef] [PubMed]

22. Carey, L.A.; Rugo, H.S.; Marcom, P.K.; Mayer, E.L.; Esteva, F.J.; Ma, C.X.; Liu, M.C.; Storniolo, A.M.; Rimawi, M.F.; Forero-Torres, A.; et al. TBCRC 001: Randomized phase II study of cetuximab in combination with carboplatin in stage IV triple-negative breast cancer. J. Clin. Oncol. 2012, 30, 2615-2623. [CrossRef] [PubMed]

23. Hynes, N.E.; Lane, H.A. ERBB receptors and cancer: The complexity of targeted inhibitors. Nat. Rev. Cancer 2005, 5, 341-354. [CrossRef] [PubMed] 
24. Zhang, H.; Berezov, A.; Wang, Q.; Zhang, G.; Drebin, J.; Murali, R.; Greene, M.I. ErbB receptors: From oncogenes to targeted cancer therapies. J. Clin. Investig. 2007, 117, 2051-2058. [CrossRef] [PubMed]

25. Canonici, A.; Ibrahim, M.F.K.; Fanning, K.; Cremona, M.; Morgan, C.; Hennessy, B.; Solca, F.; Crown, J.; O'Donovan, N. Biomarkers for afatinib and dasatinib treatment in triple negative breast cancer. Ann. Oncol. 2016, 27, 110P. [CrossRef]

26. Mullooly, M.; Conklin, D.; McGowan, P.M.; O’Brien, N.A.; O’Donovan, N.; Slamon, D.J.; Crown, J.; Finn, R.S.; Duffy, M.J. Neratinib to inhibit the growth of triple-negative breast cancer cells. J. Clin. Oncol. 2015, 33, 1099.

27. Schuler, M.; Awada, A.; Harter, P.; Canon, J.L.; Possinger, K.; Schmidt, M.; De Greve, J.; Neven, P.; Dirix, L.; Jonat, W.; et al. A phase II trial to assess efficacy and safety of afatinib in extensively pretreated patients with HER2-negative metastatic breast cancer. Breast Cancer Res. Treat. 2012, 134, 1149-1159. [CrossRef] [PubMed]

28. Ikediobi, O.N.; Davies, H.; Bignell, G.; Edkins, S.; Stevens, C.; O’Meara, S.; Santarius, T.; Avis, T.; Barthorpe, S.; Brackenbury, L.; et al. Mutation analysis of 24 known cancer genes in the NCI-60 cell line set. Mol. Cancer Ther. 2006, 5, 2606-2612. [CrossRef]

29. Yao, Z.; Torres, N.M.; Tao, A.; Gao, Y.; Luo, L.; Li, Q.; de Stanchina, E.; Abdel-Wahab, O.; Solit, D.B.; Poulikakos, P.I.; et al. BRAF Mutants Evade ERK-Dependent Feedback by Different Mechanisms that Determine Their Sensitivity to Pharmacologic Inhibition. Cancer Cell 2015, 28, 370-383. [CrossRef]

30. Misale, S.; Yaeger, R.; Hobor, S.; Scala, E.; Janakiraman, M.; Liska, D.; Valtorta, E.; Schiavo, R.; Buscarino, M.; Siravegna, G.; et al. Emergence of KRAS mutations and acquired resistance to anti-EGFR therapy in colorectal cancer. Nature 2012, 486, 532-536. [CrossRef]

31. Kumar, S.S.; Price, T.J.; Mohyieldin, O.; Borg, M.; Townsend, A.; Hardingham, J.E. KRAS G13D Mutation and Sensitivity to Cetuximab or Panitumumab in a Colorectal Cancer Cell Line Model. Gastrointest. Cancer Res. 2014, 7, 23-26. [PubMed]

32. Samatar, A.A.; Poulikakos, P.I. Targeting RAS-ERK signalling in cancer: Promises and challenges. Nat. Rev. Drug Discov 2014, 13, 928-942. [CrossRef] [PubMed]

33. McCubrey, J.A.; Steelman, L.S.; Chappell, W.H.; Abrams, S.L.; Franklin, R.A.; Montalto, G.; Cervello, M.; Libra, M.; Candido, S.; Malaponte, G.; et al. Ras/Raf/MEK/ERK and PI3K/PTEN/Akt/mTOR cascade inhibitors: How mutations can result in therapy resistance and how to overcome resistance. Oncotarget 2012, 3, 1068-1111. [CrossRef] [PubMed]

34. Sanchez-Munoz, A.; Gallego, E.; de Luque, V.; Perez-Rivas, L.G.; Vicioso, L.; Ribelles, N.; Lozano, J.; Alba, E. Lack of evidence for KRAS oncogenic mutations in triple-negative breast cancer. BMC Cancer 2010, 10, 136. [CrossRef] [PubMed]

35. Qiao, M.; Sheng, S.; Pardee, A.B. Metastasis and AKT activation. Cell Cycle 2008, 7, 2991-2996. [CrossRef]

36. Guerrero-Zotano, A.; Mayer, I.A.; Arteaga, C.L. PI3K/AKT/mTOR: Role in breast cancer progression, drug resistance, and treatment. Cancer Metastasis Rev. 2016, 35, 515-524. [CrossRef] [PubMed]

37. Heerboth, S.; Housman, G.; Leary, M.; Longacre, M.; Byler, S.; Lapinska, K.; Willbanks, A.; Sarkar, S. EMT and tumor metastasis. Clin. Transl. Med. 2015, 4, 6. [CrossRef]

38. Shibue, T.; Weinberg, R.A. EMT, CSCs, and drug resistance: The mechanistic link and clinical implications. Nat. Rev. Clin. Oncol 2017, 14, 611. [CrossRef]

39. Kim, S.B.; Dent, R.; Im, S.A.; Espie, M.; Blau, S.; Tan, A.R.; Isakoff, S.J.; Oliveira, M.; Saura, C.; Wongchenko, M.J.; et al. Ipatasertib plus paclitaxel versus placebo plus paclitaxel as first-line therapy for metastatic triple-negative breast cancer (LOTUS): A multicentre, randomised, double-blind, placebo-controlled, phase 2 trial. Lancet Oncol. 2017, 18, 1360-1372. [CrossRef]

40. Van Sebille, Y.Z.A.; Gibson, R.J.; Wardill, H.R.; Bowen, J.M. ErbB small molecule tyrosine kinase inhibitor (TKI) induced diarrhoea: Chloride secretion as a mechanistic hypothesis. Cancer Treat. Rev. 2015, 41, 646-652. [CrossRef]

41. Liu, C.Y.; Huang, T.T.; Chu, P.Y.; Huang, C.T.; Lee, C.H.; Wang, W.L.; Lau, K.Y.; Tsai, W.C.; Chao, T.I.; $\mathrm{Su}$, J.C.; et al. The tyrosine kinase inhibitor nintedanib activates SHP-1 and induces apoptosis in triple-negative breast cancer cells. Exp. Mol. Med. 2017, 49, e366. [CrossRef] [PubMed]

(C) 2019 by the authors. Licensee MDPI, Basel, Switzerland. This article is an open access article distributed under the terms and conditions of the Creative Commons Attribution (CC BY) license (http://creativecommons.org/licenses/by/4.0/). 